\title{
Early diphtheria-tetanus-pertussis vaccination associated with higher female mortality and no difference in male mortality in a cohort of low birthweight children: an observational study within a randomised trial
}

\author{
Peter Aaby, ${ }_{1}$ Henrik Ravn, ${ }^{2}$ Adam Roth, ${ }^{2,3}$ Amabelia Rodrigues, ${ }^{1}$ Ida Maria Lisse, ${ }^{4}$ \\ Birgitte Rode Diness, ${ }^{1}$ Karen Rokkedal Lausch, ${ }^{1}$ Najaaraq Lund, ${ }^{1}$ Julie Rasmussen, \\ Sofie Biering-Sørensen, ${ }^{1}$ Hilton Whittle, ${ }^{5}$ Christine Stabell Benn ${ }^{2}$
}

- An additional appendix is published online only. To view this file please visit the journal online (http://adc.bmj.com/ content/early/recent)

'Bandim Health Project, Bissau, Guinea-Bissau 2Bandim Health Project, Statens Serum Institut, Copenhagen, Denmark ${ }^{3}$ Department of Medical Microbiology, Lund University, Malmö, Sweden

${ }^{4}$ Department of Pathology, Herlev University Hospital, Copenhagen, Denmark ${ }^{5}$ MRC Laboratories, Fajara, Gambia

\section{Correspondence to} Peter Aaby, Bandim Health Project, Statens Serum Institut, Artillerivej 5, 2300 Copenhagen S, Denmark; p.aaby@bandim.org

Received 6 July 2011 Accepted 18 January 2012 Published Online First 13 February 2012

\section{SUMMARY}

Background Studies from low-income countries have suggested that diphtheria-tetanus-pertussis (DTP) vaccine provided after Bacille Calmette-Guerin (BCG) vaccination may have a negative effect on female survival. The authors examined the effect of DTP in a cohort of low birthweight (LBW) infants.

Methods 2320 LBW newborns were visited at 2, 6 and 12 months of age to assess nutritional and vaccination status. The authors examined survival until the 6-month visit for children who were DTP vaccinated and DTP unvaccinated at the 2-month visit.

Results Two-thirds of the children had received DTP at 2 months and 50 deaths occurred between the 2-month and 6-month visits. DTP vaccinated children had a better anthropometric status for all indices than DTP unvaccinated children. Small mid-upper arm circumference (MUAC) was the strongest predictor of mortality. The death rate ratio (DRR) for DTP vaccinated versus DTP unvaccinated children differed significantly for girls (DRR 2.45; 95\% Cl 0.93 to 6.45 ) and boys (DRR $0.53 ; 95 \% \mathrm{Cl} 0.23$ to 1.20$)(p=0.018$, homogeneity test). Adjusting for MUAC, the overall effect for DTP vaccinated children was 2.62 (95\% Cl 1.34 to 5.09$)$; DRR was 5.68 (95\% Cl 1.83 to 17.7) for girls and 1.29 (95\% Cl 0.56 to 2.97) for boys ( $p=0.023$, homogeneity test). While anthropometric indices were a strong predictor of mortality among boys, there was little or no association for girls.

Conclusion Surprisingly, even though the children with the best nutritional status were vaccinated early, early DTP vaccination was associated with increased mortality for girls.

\section{INTRODUCTION}

Ten years ago we reported that Bacille CalmetteGuerin (BCG) and diphtheria-tetanus-pertussis (DTP) vaccines surprisingly had opposite effects on child survival in Guinea-Bissau. ${ }^{12}$ BCG was associated with a $45 \%$ reduction in mortality over the following 6 months, whereas early DTP was associated with an $84 \%$ increase in mortality. The negative effect of DTP was strongest for girls. ${ }^{2}$ In response, WHO sponsored several groups to reanalyse existing data, but all still reported that

\section{What is already known on this topic}

- Live vaccines such as measles vaccine and Bacille Calmette-Guerin have non-specific beneficial effects in areas with high mortality.

- Previous studies from several low-income African countries have suggested that inactivated vaccines, including diphtheriatetanus-pertussis (DTP), may have nonspecific negative effects for survival of girls.

- WHO sponsored studies which have found a beneficial effect of DTP have major methodological problems.

\section{What this study adds}

- Adjusted for nutritional status, DTP vaccinated children, particularly girls, had threefold higher mortality between 2 and 6 months of age.

- Nutritional status was a strong predictor of mortality among boys but not girls.

- There is a continuing contradiction between studies of DTP from low-income countries and current policy.

DTP had major beneficial effects. ${ }^{3-8}$ In contrast, we have continued to find negative effects for DTP for girls in Gambia ${ }^{9}$, Senegal, ${ }^{10}$ Ghana, ${ }^{11}$ Sudan, ${ }^{12}$ Congo $^{12}$ and Malawi. ${ }^{13}$

There are methodological and contextual problems behind these contrasting results. First, we used a landmark approach: children were included in the survival analysis from the date the vaccination card was seen. ${ }^{14}$ Most WHO sponsored studies used a retrospective updating approach: children were included in the survival analysis from the vaccination date detected retrospectively. ${ }^{3-8}$ This leads to survival bias; if information is better for survivors than for dead children, then vaccinated children who have died will be misclassified as unvaccinated, exaggerating the beneficial effect of vaccines. ${ }^{14-16}$ Second, we evaluated children who followed the WHO recommended schedule online under the BMJ Journals unlocked scheme, see http:// ard.bmj.com/info/unlocked.dt| 
of BCG at birth and DTP at 6 weeks. However, in most WHO sponsored studies, two-thirds or more had received BCG and DTP simultaneously. ${ }^{3} 467$

The uncertainty created by the contrasting results on DTP needs to be resolved. We have previously formulated the hypothesis that DTP vaccination is associated with substantially higher mortality in girls. ${ }^{16}$ We tested the hypothesis using data from a low birthweight (LBW) cohort. ${ }^{17} 18$

\section{METHODS}

\section{Setting}

The Bandim Health Project (BHP) in Guinea-Bissau runs a health and demographic surveillance system in the capital covering approximately 102000 people, around 30\% of the population in the capital and $6 \%$ of the population in the country. Children less than 3 years of age are visited every 3 months to collect information on breastfeeding, hospitalisation, vaccination status, background factors and survival. Furthermore, $\mathrm{BHP}$ registers all births at the maternity ward in Bissau and LBW babes and their mothers are driven home to facilitate follow-up.

\section{LBW cohort}

BCG is not given at birth to LBW children. The LBW cohort was initiated to study the impact of BCG at birth and neonatal vitamin $A$ on infant mortality in a randomised trial (ClinicalTrials.gov NCT00146302 and ClinicalTrials.gov NCT00168610). ${ }^{17} 18$ Since twins weigh less than singletons, a large proportion of LBW cohorts are twins. LBW children were enrolled at the maternity ward or at the first health centre contact after birth. We used the data on routine vaccinations to study the impact of DTP on mortality between the 2-month and 6-month visits.

\section{Information on vaccinations}

At each visit, weight, length, arm circumference, head circumference and abdominal circumference were measured and a tuberculin skin test carried out. Importantly, it was also registered whether the vaccination card was seen and all vaccine dates were noted. Vaccinations after the 2-month visit were not used in the survival analysis as this would have introduced survival bias. A total of 13 children received measles vaccine (MV) at $4 \frac{1}{2}-5$ months of age as part of a trial of early MV. ${ }^{19}$ We also asked to see the vaccination cards of children who had died.

\section{Child deaths}

Around 3 months after a death, a verbal autopsy was conducted by a clinician who assigned a probable diagnosis.

\section{Statistical analysis}

The main outcome was the effect of early DTP (yes/no) on mortality analysed in a Cox proportional hazards model with age as underlying time. Children entered at 2 months of age and were followed to death, measles vaccination, migration or the 6-month visit, whichever came first. We adjusted for possible dependence of life-times of twins by calculating standard errors that allow for intra-twin correlation (robust standard

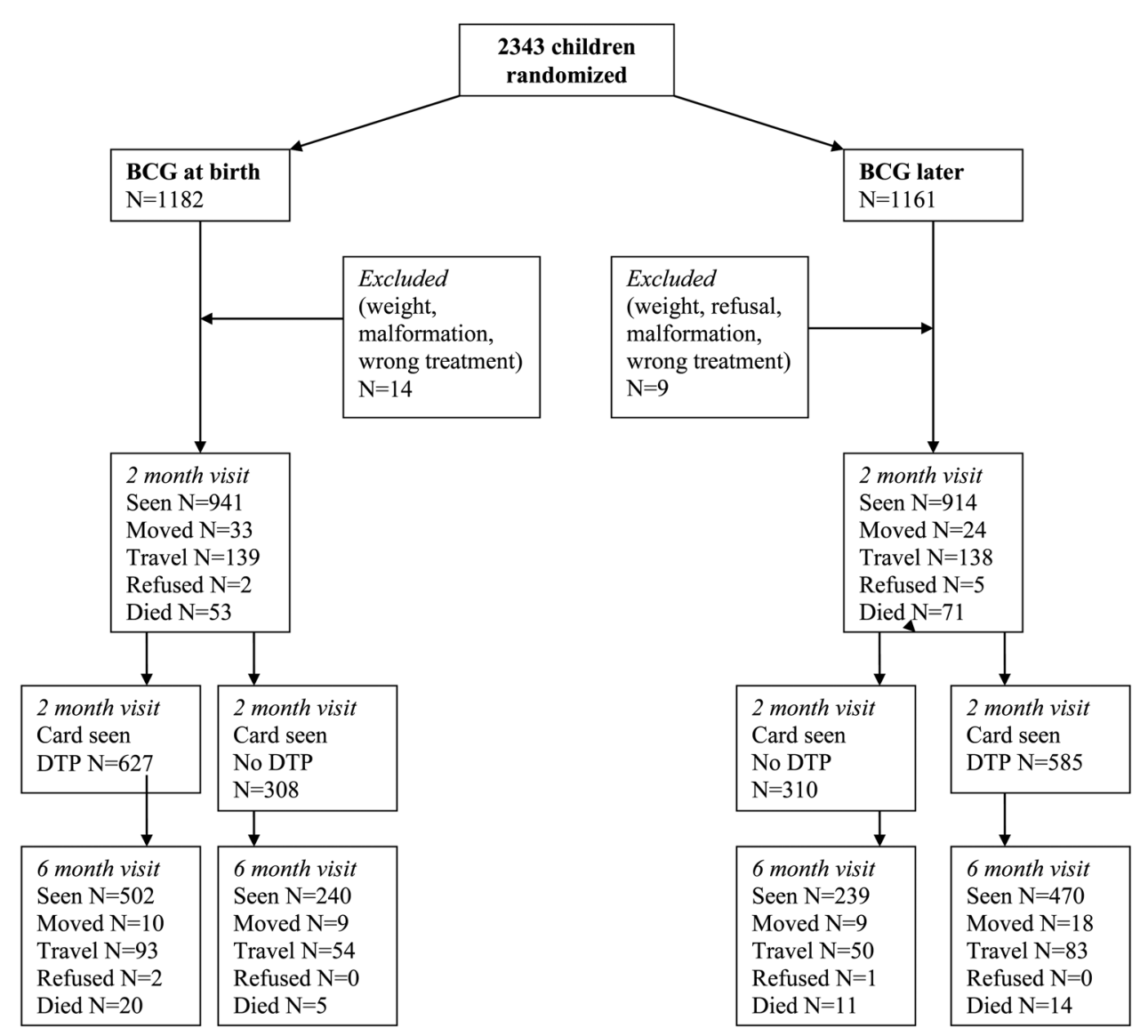

Figure 1 Study profile. BCG, Bacille Calmette-Guerin vaccine; DTP, diphtheria-tetanus-pertussis vaccine. 
errors and $95 \%$ CIs) as $22 \%$ of the children were twins. We conducted a secondary analysis in which children were followed to 9 months of age, the age of routine measles vaccination; this analysis is less certain because date of death was collected several months later and some children may have been vaccinated against measles before 9 months unbeknownst to us.

The randomisation to BCG at birth or later influenced the sequence of vaccinations and all analyses were therefore adjusted for the BCG arm. The results are presented as death rate ratios (DRR) with $95 \%$ CIs. The assumption of proportionality in the Cox model was checked using Schoenfeld residuals ( $p>0.20$, for both sex-specific DTP effects adjusting for BCG arm and mid-upper arm circumference (MUAC)). The effects of anthropometric indices were modelled as quantitative variables expressing a change in mortality per unit increase of the index. We tested for log-linearity of quantitative variables (eg, weight) using a quadratic factor. We used a 'homogeneity test' to represent a test of no interaction. The Kaplan-Meier method was used to draw crude cumulated mortality curves. We assessed nutritional status by anthropometric measurements. We calculated weight-for-age $z$ scores and height-forage $z$ scores using WHO child growth standards (http://www. who.int/childgrowth/software/). The weight-for-height $z$ score (WHZ) had too many missing values $(\mathrm{N}=29)$ because the programme does not calculate scores for children with a height of less than $45 \mathrm{~cm}$. Results for WHZ have therefore not been presented. Nutritional status influenced time to DTP vaccination and we therefore controlled for a healthy-vaccinee effect by adjusting for anthropometric measurements.

\section{RESULTS}

\section{The LBW cohort}

The follow-up in the LBW cohort is described in figure 1. Overall, 1855 children were seen at home at the 2 -month visit and their median age was 65 days. It was possible to see the vaccination card for 1830 children (99\%) and only these children were included in the analyses of the effect of DTP on survival (figure 1).

\section{DTP vaccination status}

There were 618 (34\%) DTP unvaccinated children at 2 months of age, called the delayed DTP group in the present analysis.
Among the 1212 DTP vaccinated children, the mean age of vaccination was 51 days for both sexes. The median interval between the date of BCG vaccination and DTP vaccination was 46 days (25-75 percentiles: $44-51$ days) in the BCG at birth group, whereas it was 0 days (25-75 percentiles: 0-19 days) in the delayed BCG arm. In the delayed BCG arm, 38\% (159/421) received BCG before DTP, 53\% (225/421) BCG and DTP simultaneously and 9\% (37/421) BCG after DTP. The delayed DTP group had significantly lower anthropometric indices (table 1), suggesting that the healthiest children received DTP early.

Several variables differed between the groups (table 2). Mothers of delayed DTP children were younger, had less schooling and more children were twins. There were fewer unvaccinated children in Bandim where children are actively called for vaccination. Normally all children are breastfed to at least 12 months of age. However, the non-governmental organisation responsible for preventing maternal HIV transmission recommended infected mothers not to breastfeed. The proportion of non-breastfeeding children was similar in the two groups, suggesting that the proportion of HIV infected mothers was the same. The differences in health and socioeconomic indicators were similar for boys and girls (data not shown).

\section{Vaccinations between 2 and 6 months of age}

The rate of DTP vaccination between the 2 -month and 6-month visits was higher in the early DTP group (1040 vaccinations per 43672 observation days) than in the delayed DTP group (379 vaccinations per 42771 observation days), the age-adjusted incidence rate ratio (IRR) being 2.28 (95\% CI 2.01 to 2.60$)$. The pattern was similar for boys (IRR 2.42 ; $95 \%$ CI 2.01 to 2.91 ) and girls (IRR 2.18 ; $95 \%$ CI 1.85 to 2.58 ). Among DTP unvaccinated children seen at the 6-month visit, 69\% (323/471) had received DTP, the median age being 99 days (25-75 quartiles: 78-133). Of the 16 initially DTP unvaccinated children (five girls, 11 boys) who died between 2 and 6 months, at least five (three girls, two boys) received DTP before they died.

\section{Mortality}

By the 6 -month visit, 50 children had died (2.7\%) (figure 1$)$. The effect of DTP (table 3 ) differed significantly for girls and boys (figure 2). The DRR for early versus delayed DTP vaccination

Table 1 Health indicators for DTP vaccinated and DTP unvaccinated children at the 2-month visit

\begin{tabular}{|c|c|c|c|}
\hline Variable & $\begin{array}{l}\text { DTP unvaccinated } \\
N=618\end{array}$ & $\begin{array}{l}\text { DTP vaccinated } \\
\mathrm{N}=1212\end{array}$ & $\begin{array}{l}\text { Difference for vaccinated compared } \\
\text { with unvaccinated }(95 \% \mathrm{CI})\end{array}$ \\
\hline Median age at visit (boys), days & 64 & 66 & $\mathrm{p}<0.001$ (Kruskal-Wallis) \\
\hline Median age at visit (girls), days & 65 & 65 & $p=0.152$ \\
\hline Mean maternal MUAC, mm & 236 & 241 & $5.1(2.4$ to 7.8$)$ \\
\hline Mean Ballard score at birth & $35(N=585)$ & $37(N=1073)$ & $2.4(1.8$ to 3.0$)$ \\
\hline Mean birth weight, kg & 2.060 & 2.200 & $\mathrm{p}<0.001$ (Kruskal-Wallis) \\
\hline Mean weight gain between enrolment and 2-month visit, kg & 1.937 & 2.275 & $0.338(0.273$ to 0.402$)$ \\
\hline Mean weight at 2 months, $\mathrm{kg}$ & 3.901 & 4.406 & $0.505(0.426$ to 0.585$)$ \\
\hline Weight-for-age z score (SD) & $-3.1(1.7)$ & $-2.3(1.2)$ & $0.74(0.60$ to 0.88$)$ \\
\hline Mean length at 2 months, $\mathrm{cm}$ & 52 & 54 & $1.7(1.4$ to 2.0$)$ \\
\hline Height-for-age z score (SD) & $-2.7(1.6)$ & $-1.8(1.1)$ & $0.84(0.72$ to 0.97$)$ \\
\hline Mean MUAC at 2 months, mm & 106 & 115 & $8.4(6.9$ to 9.7$)$ \\
\hline Mean head circumference at 2 months, mm & 371 & 378 & $8(6$ to 9$)$ \\
\hline Mean abdominal circumference at 2 months, $\mathrm{mm}$ & 336 & 350 & $15(12$ to 18$)$ \\
\hline
\end{tabular}

Differences were similar for boys and girls except for age at enrolment.

DTP, diphtheria-tetanus-pertussis; MUAC, mid-upper arm circumference. 
Table 2 Background factors for DTP vaccinated and DTP unvaccinated children

\begin{tabular}{|c|c|c|c|}
\hline Variable & $\frac{\text { DTP unvaccinated }}{\mathrm{N}=618}$ & $\frac{\text { DTP vaccinated }}{\mathrm{N}=1212}$ & $\begin{array}{l}\text { RR for vaccinated compared } \\
\text { with unvaccinated }(95 \% \mathrm{CI})\end{array}$ \\
\hline Mother's age: $13-19$ years & $33 \%(198 / 603)$ & $27 \%(320 / 1190)$ & $0.82(0.71$ to 0.95$)$ \\
\hline Living with father of child & $63 \%(388 / 612)$ & $59 \%(716 / 1209)$ & $0.93(0.87$ to 1.01$)$ \\
\hline Mother's ethnic group: Pepel, Manjaco or Mancanha & $26 \%(159 / 613)$ & $32 \%(384 / 1207)$ & $1.23(1.05$ to 1.44$)$ \\
\hline Mother's schooling: none & $44 \%(270 / 616)$ & $31 \%(390 / 1210)$ & $0.74(0.65$ to 0.83$)$ \\
\hline Mother died & $0.5 \%(3 / 618)$ & $0.3 \%(4 / 1212)$ & $0.86(0.45$ to 1.64$)$ \\
\hline From BHP study area & $17 \%(104 / 618)$ & $37 \%(443 / 1212)$ & 1.35 (1.27 to 1.44$)$ \\
\hline Child is firstborn & $43 \%(268 / 618)$ & $46 \%(554 / 1212)$ & $1.05(0.95$ to 1.18$)$ \\
\hline Boys & $42 \%(258 / 618)$ & $44 \%(539 / 1212)$ & 1.04 (0.97 to 1.11$)$ \\
\hline Twins & $27 \%(168 / 618)$ & $19 \%(235 / 1212)$ & $0.85(0.78$ to 0.93$)$ \\
\hline Not breastfeeding at 2-month visit* & $2 \%(14 / 618)$ & $2 \%(28 / 1211)$ & $0.99(0.80$ to 1.23$)$ \\
\hline Consultations before visit & $43 \%(263 / 616)$ & $43 \%(516 / 1201)$ & 1.00 (0.94 to 1.07$)$ \\
\hline Received BCG vaccine before 2-month visit & $68 \%(420 / 618)$ & $86 \%(1048 / 1212)$ & $1.58(1.40$ to 1.77$)$ \\
\hline Randomised to BCG at birth & $50 \%(308 / 618)$ & $52 \%(627 / 1212)$ & $1.03(0.96$ to 1.10$)$ \\
\hline Randomised to vitamin A at birth & $51 \%(244 / 481)$ & $49 \%(439 / 895)$ & $0.98(0.90$ to 1.06$)$ \\
\hline Positive tuberculin skin test reaction & $9 \%(54 / 576)$ & $11 \%(121 / 1146)$ & $1.04(0.94$ to 1.16$)$ \\
\hline
\end{tabular}

* If a child was not breastfed at 2 months of age it usually meant that the mother had died or was HIV infected.

BCG, Bacille Calmette-Guerin; BHP, The Bandim Health Project; DTP, diphtheria-tetanus-pertussis.

The values in parentheses in the second and third columns denote factor/observed.

was 2.45 (95\% CI 0.93 to 6.45) for girls and 0.53 (95\% CI 0.23 to 1.20$)$ for boys ( $p=0.018$, test of homogeneity), suggesting a negative effect for girls and a protective effect for boys. We conducted additional analyses controlling for the health and socio-economic indicators in tables 1 and 2, including birth weight, study area, sex, twinning, birth order, breastfeeding status, MUAC, consultations, maternal MUAC, mother's age, ethnic group, mother's schooling, living with the father, randomisation to BCG and randomisation to vitamin A. In this analysis excluding 152 children and four deaths due to missing observations, the DRR for DTP vaccinated versus the delayed DTP group was 2.55 (95\% CI 0.92 to 7.11) for girls and 0.36 (95\% CI 0.14 to 0.89$)$ for boys $(p=0.004)$. DTP had similar effects among children who had received vitamin $\mathrm{A}$ at birth and those who had not. ${ }^{18}$ In the early DTP group, age at vaccination or time between DTP vaccination and the 2 -month visit did not affect subsequent mortality (data not shown). Verbal autopsy did not suggest major differences in causes of death between sex or vaccine groups (see online supplementary appendix table).

We adjusted for nutritional status to control for the healthy vaccinee effect (table 3 ). In multiple Cox regression analyses, MUAC was the main determinant although all anthropometric indices were strongly associated with mortality if MUAC was not included. Adjusted for MUAC at the 2-month visit, the DRR for the early versus delayed DTP group was $2.62(95 \%$ CI 1.34 to 5.09$)$, being 5.68 (95\% CI 1.83 to 17.7$)$ for girls and 1.29 (95\% CI 0.56 to 2.97 ) for boys (table 3 ) ( $p=0.023$, test of homogeneity). With follow-up to 9 months of age, the DRR was 4.46 (95\% CI 1.55 to 12.8 ) for girls and 1.28 (95\% CI 0.61 to 2.68 ) for boys ( $p=0.043$ ). Adjustment for weight-for-age $z$ scores produced essentially similar results, the DRR being 5.37 (95\% CI 1.71 to 16.9 ) for girls and 1.16 (95\% CI 0.50 to 2.72 ) for boys ( $p=0.019)$. Adjusting for height-for-age $z$ scores, the DRR was 3.26 (95\% CI 1.17 to 9.07) for girls and 0.75 (95\% CI 0.33 to 1.69$)$ for boys $(p=0.022)$.

The negative effect for girls was similar whether they had been DTP vaccinated shortly before the 2 -month visit or earlier: the DRR was 5.79 (95\% CI 1.39 to 24.2) for those vaccinated within 7 days and 6.25 (95\% CI 1.87 to 20.0) for those vaccinated within 14 days of the 2 -month visit.
The negative effect of DTP was stronger in the BCG arm (DRR 4.33; 95\% CI 1.54 to 12.2) than in the delayed BCG arm (DRR 1.71; 95\% CI 0.73 to 4.01$)(p=0.147)$ (table 3). The negative effect for girls was seen in both the BCG arm (DRR 7.18; 95\% CI 1.53 to 33.7) and the delayed BCG arm (DRR 4.26; $95 \%$ CI 0.96 to 18.9$)$.

\section{Nutritional status of children who died}

For boys, all nutritional indices at the 2 -month visit were significantly associated with mortality between the 2-month and 6-month visits (table 4). However, for girls, most of these indices were not significantly associated with mortality (table 4). For all seven nutritional indices, the association was significantly different for boys and girls (tests of homogeneity). For boys the effect was similar in the early and delayed DTP groups; for girls the pattern could only be assessed for the early DTP group as there were too few deaths in the delayed DTP group.

\section{DISCUSSION}

\section{Main observations}

First, the selection bias for vaccination was very strong, with the healthier children receiving DTP earlier. Second, DTP was associated with a significantly different effect on mortality for boys and girls in both crude and adjusted analyses. Third, in adjusted analyses DTP vaccinated girls had significantly higher mortality than girls in the delayed DTP group. Fourth, the negative effect of early DTP was most marked for children randomised to the WHO schedule of BCG at birth and then DTP 6-7 weeks later. Fifth, nutritional status had no or limited impact on survival among girls, whereas the association with survival was very strong among boys.

\section{Strengths and weaknesses}

We resolved the methodological problems found in previous studies of DTP.14 152021 First, classification as 'unvaccinated' was based on reading the vaccination card and did not include children with 'no information'. ${ }^{15}$ Second, limiting the analyses to the 2-6-month or 2-9-month periods made it unlikely that results were confounded by subsequent measles vaccination. 
Table 3 DRR between 2 and 6 months of age according to initial vaccination status and sex

\begin{tabular}{|c|c|c|c|c|c|c|c|c|c|c|}
\hline \multirow[b]{2}{*}{ Sex } & \multicolumn{4}{|c|}{$\begin{array}{c}\text { Mortality per } 100 \text { person-years (deaths/days } \\
\text { of observation) }\end{array}$} & \multicolumn{3}{|c|}{ Crude DRR (95\% CI) (DTP/no DTP) } & \multicolumn{3}{|c|}{ DRR (95\% CI) (DTP/no DTP) adjusted* } \\
\hline & $\begin{array}{l}\text { DTP } \\
\text { vaccinated }\end{array}$ & No DTP & $\begin{array}{l}\text { DTP } \\
\text { vaccinated }\end{array}$ & No DTP & BCG at birth & Delayed BCG & All & BCG at birth & Delayed BCG & All \\
\hline Boys & $\begin{array}{l}8.0 \\
(7 / 31885)\end{array}$ & $\begin{array}{l}6.8 \\
(3 / 16161)\end{array}$ & $\begin{array}{l}5.9 \\
(5 / 30952)\end{array}$ & $\begin{array}{l}20.3 \\
(8 / 14415)\end{array}$ & $\begin{array}{l}1.18 \\
(0.30 \text { to } 4.55)\end{array}$ & $\begin{array}{l}0.29 \\
(0.10 \text { to } 0.89)\end{array}$ & $\begin{array}{l}0.53 \\
(0.23 \text { to } 1.20)\end{array}$ & $\begin{array}{l}2.48 \\
(0.61 \text { to } 10.0)\end{array}$ & $\begin{array}{l}0.79 \\
(0.26 \text { to } 2.38)\end{array}$ & $\begin{array}{l}1.29 \\
\text { (0.56 to } 2.97)\end{array}$ \\
\hline Girls & $\begin{array}{l}11.5 \\
(13 / 41361)\end{array}$ & $\begin{array}{l}3.4 \\
(2 / 21194)\end{array}$ & $\begin{array}{l}8.8 \\
(9 / 37358)\end{array}$ & $\begin{array}{l}4.8 \\
(3 / 22656)\end{array}$ & $\begin{array}{l}3.34 \\
(0.76 \text { to } 14.6)\end{array}$ & $\begin{array}{l}1.81 \\
(0.49 \text { to } 6.69)\end{array}$ & $\begin{array}{l}2.45 \\
(0.93 \text { to } 6.45)\end{array}$ & $\begin{array}{l}7.18 \\
\text { (1.53 to } 33.7)\end{array}$ & $\begin{array}{l}4.26 \\
(0.96 \text { to } 18.9)\end{array}$ & $\begin{array}{l}5.68 \\
(1.83 \text { to } 17.7)\end{array}$ \\
\hline All & $\begin{array}{l}10.0 \\
(20 / 73246)\end{array}$ & $\begin{array}{l}4.9 \\
(5 / 37355)\end{array}$ & $\begin{array}{l}7.5 \\
(14 / 68310)\end{array}$ & $\begin{array}{l}10.8 \\
(11 / 37071)\end{array}$ & $\begin{array}{l}2.04 \\
\text { (0.77 to } 5.41)\end{array}$ & $\begin{array}{l}0.68 \\
(0.31 \text { to } 1.51)\end{array}$ & $\begin{array}{l}1.11 \\
(0.61 \text { to } 2.02)\end{array}$ & $\begin{array}{l}4.33 \\
(1.54 \text { to } 12.2)\end{array}$ & $\begin{array}{l}1.71 \\
(0.73 \text { to } 4.01)\end{array}$ & $\begin{array}{l}2.62 \\
\text { (1.34 to } 5.09)\end{array}$ \\
\hline
\end{tabular}

${ }^{*}$ Adjusted for arm of BCG trial, sex and arm circumference.

BCG, Bacille Calmette-Guerin; DRR, death rate ratios; DTP, diphtheria-tetanus-pertussis.
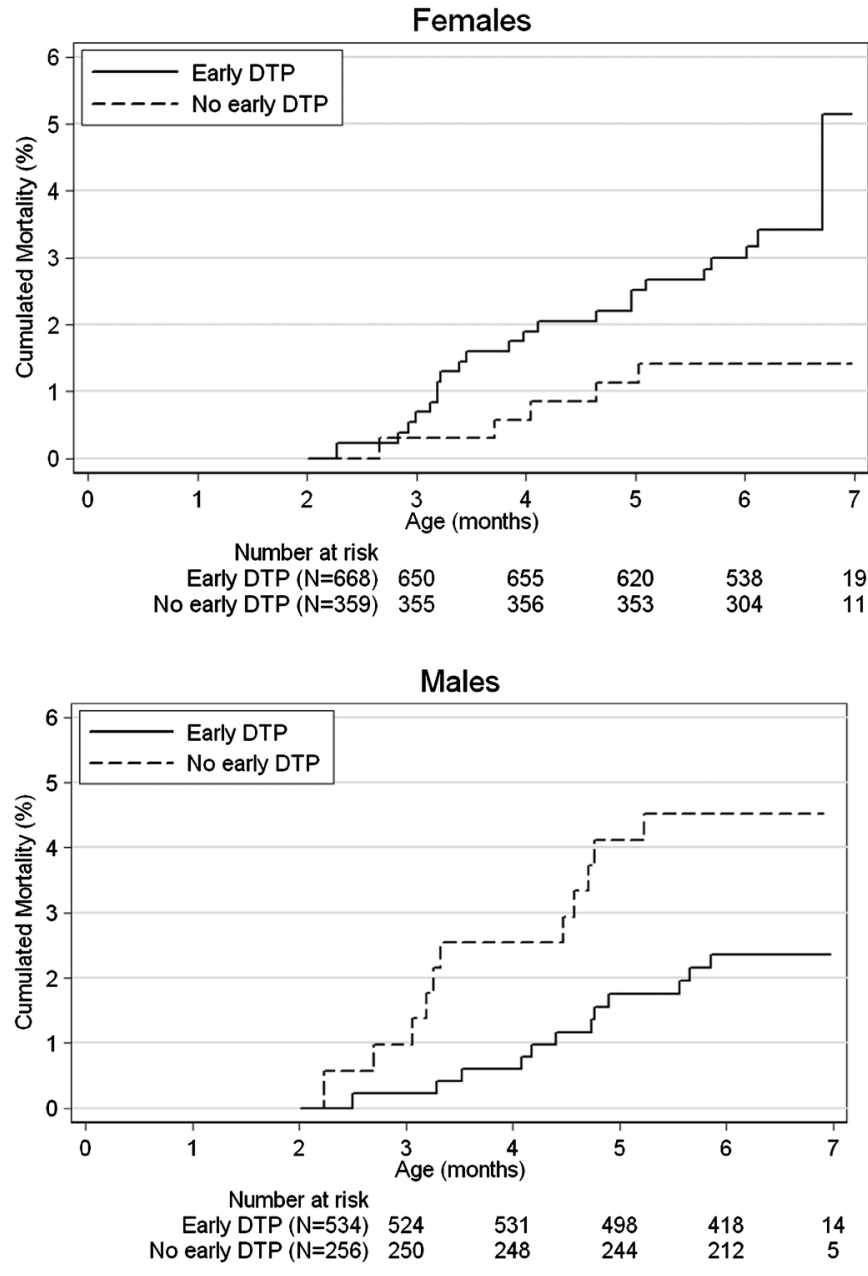

Figure 2 Accumulated mortality curves for DTP vaccinated and not yet DTPvaccinated children at 2 months of age for females and males. As $1 \%$ of the children had the 2-month visit after 3 months of age, the number at risk increased between 3 and 4 months of age. DTP, diphtheria-tetanus-pertussis vaccine.

Third, follow-up was very good for a highly mobile urban population. Fourth, we could control for many nutritional, health and socio-economic indicators.

The effect of early DTP did not depend on the timing of DTP vaccination. We focused on the 2-6-month period for which the data quality would be best, but the negative effect remained the same until 9 months of age when the children received MV. Only half the children followed the WHO schedule of BCG at birth and DTP later. Since this group displayed the strongest negative effect, we may have underestimated the negative effect of DTP.

\section{Interpretation}

Comparing subsequent mortality for DTP vaccinated and DTP unvaccinated children at the 2 -month visit is not equivalent to testing DTP vaccination versus no DTP vaccination because more than two-thirds of the unvaccinated children received DTP during follow-up. However, this comparison measures the effect of early DTP vaccination. Since the healthiest children were vaccinated early, DTP vaccinated children should have had lower mortality than the delayed DTP group. It has been suggested that sick children come to the health centre for treatment and receive DTP vaccination simultaneously and that this could explain why DTP vaccination is associated with increased mortality. ${ }^{42}$ That is not the case in Bissau. The children are coming to the health centre either for treatment or vaccination.

The crude and adjusted analyses gave similar results. We see no selection bias which can explain why DTP has different effects for boys and girls or the higher mortality among DTP vaccinated girls. Sex-preferential treatment cannot explain this pattern. After measles vaccination girls have lower mortality than boys. Furthermore, in the present study (table 3), girls had higher mortality than boys among DTP vaccinated children but lower mortality than boys in the delayed DTP group. The DTP related excess mortality was not seen merely among malnourished girls at risk of death. Hence, DTP may deregulate the female immune system so that subsequent unrelated infections are fought inefficiently. ${ }^{23} 24$ There are few studies of immunological differences between boys and girls in response to vaccination. Females are more Th2-prone than males in animal models ${ }^{25}$ and DTP stimulates a Th2 response. ${ }^{26}$ In animal models, a Th2 response is dysfunctional. ${ }^{25} 26$ Furthermore, DTP may affect retinol levels ${ }^{27}$ and maternal antibodies differentially for boys and girls ${ }^{28}$ making girls susceptible to infections earlier. ${ }^{29}$ Previous studies suggest that the negative effect of DTP applies to all major types of fatal childhood infections in low-income countries. ${ }^{3031}$

\section{Consistency with previous studies}

The negative effect of DTP found in the present study corroborates previous reports. ${ }^{19} 1030-35$ DTP is associated with higher female mortality whether it is administered before or after 
Table 4 Differences in nutritional status between children who died and those who survived according to sex

\begin{tabular}{|c|c|c|c|}
\hline \multirow[b]{2}{*}{$\begin{array}{l}\text { Anthropometric indices } \\
\text { at } 2 \text { months of age }\end{array}$} & \multicolumn{3}{|c|}{ Median anthropometric indices (number of children) } \\
\hline & $\begin{array}{l}\text { Survived between } \\
2 \text { and } 6 \text { months of age }\end{array}$ & $\begin{array}{l}\text { Died between } 2 \text { and } \\
6 \text { months of age }\end{array}$ & $\begin{array}{l}\text { DRR ( } 95 \% \mathrm{CI} \text { ) associated with } \\
\text { one unit increase in nutritional status }\end{array}$ \\
\hline \multicolumn{4}{|l|}{ Median weight $(\mathrm{kg})$} \\
\hline Boys & $4.460(764)$ & $3.100(23)$ & 0.19 (0.11 to 0.31$), p<0.001$ \\
\hline Girls & $4.220(998)$ & $4.040(27)$ & 0.70 (0.41 to 1.19$), p=0.187$ \\
\hline \multicolumn{4}{|l|}{ Median WAZ } \\
\hline Boys & $-2.0(764)$ & $-4.21(23)$ & $0.39(0.30$ to 0.50$), \mathrm{p}<0.001$ \\
\hline Girls & $-1.69(998)$ & $-2.2(27)$ & $0.78(0.60$ to 1.01$), p=0.056$ \\
\hline \multicolumn{4}{|l|}{ Median height $(\mathrm{cm})$} \\
\hline Boys & $54.0(767)$ & $50.5(23)$ & 0.74 (0.66 to 0.83 ), $\mathrm{p}<0.001$ \\
\hline Girls & $53.0(1000)$ & $53.5(27)$ & $1.04(0.94$ to 1.16$), p=0.440$ \\
\hline \multicolumn{4}{|l|}{ Median HAZ } \\
\hline Boys & $-2.61(767)$ & $-4.21(23)$ & 0.53 (0.42 to 0.67$), p<0.001$ \\
\hline Girls & $-2.14(1000)$ & $-2.13(27)$ & $1.03(0.84$ to 1.25$), p=0.795$ \\
\hline \multicolumn{4}{|l|}{ Median MUAC (mm) } \\
\hline Boys & $116(766)$ & $86(23)$ & 0.90 (0.88 to 0.93 ), $p<0.001$ \\
\hline Girls & 112 (998) & $110(27)$ & $0.97(0.94$ to 0.99$), p=0.026$ \\
\hline \multicolumn{4}{|l|}{$\begin{array}{l}\text { Median head } \\
\text { circumference }(\mathrm{cm})\end{array}$} \\
\hline Boys & $38(766)$ & $36(23)$ & 0.57 (0.46 to 0.71$), p<0.001$ \\
\hline Girls & $37(1000)$ & $38(27)$ & 0.96 (0.79 to 1.17$), p=0.681$ \\
\hline \multicolumn{4}{|l|}{$\begin{array}{l}\text { Median abdominal } \\
\text { circumference }(\mathrm{cm})\end{array}$} \\
\hline Boys & $35(767)$ & $31(23)$ & 0.72 (0.66 to 0.80$), p<0.001$ \\
\hline Girls & $35(1000)$ & $34(27)$ & $0.86(0.76$ to 0.98$), p=0.027$ \\
\hline
\end{tabular}

Adjusted for arm of BCG trial and DTP at 2 months.

BCG, Bacille Calmette-Guerin; DRR, death rate ratios; HAZ, height-for-age z score; MUAC, mid-upper-arm-circumference; WAZ, weight-for-age $z$ score.

MV. ${ }^{911-13} 3637$ The increased female mortality after high-titre measles vaccine (HTMV), which led to the global withdrawal of HTMV, was related to DTP being administered after HTMV. ${ }^{35}$ Vitamin A supplementation amplifies the negative effect of DTP for girls. ${ }^{11} 18$ 38-40 Furthermore, we conducted two randomised trials providing a live vaccine shortly after DTP vaccination, thereby reducing exposure to DTP as the most recent vaccination. First, we provided MV at 4 months shortly after DTP ${ }^{41}$ and second, BCG revaccination after booster DTP. ${ }^{42}$ The effect was beneficial in both trials. Hence, DTP may induce an harmful immune profile that live vaccines can improve.

\section{Implications}

The negative effect of DTP has only been studied in situations with herd immunity to pertussis. Little is known about pertussis-related child mortality in the prevaccination era in Africa. Only one community study in Kenya has assessed the impact of pertussis: in the early phase of the vaccination programme, case fatality was $1.3 \%$ and pertussis accounted for $6 \%$ of infant deaths. ${ }^{43}$ With increases in mortality of $80-150 \%$ in observational studies ${ }^{15} 3233$ and threefold reductions in mortality in randomised trials providing a live vaccine shortly after DTP vaccination and thereby reducing exposure to DTP as the most recent vaccination, ${ }^{41}{ }^{42}$ DTP is likely associated with increased female mortality also in situations without herd immunity.

The inconsistency between the evidence and current policy is unacceptable. The Global Advisory Committee on Vaccine Safety has indicated that it will monitor the non-specific effects of vaccines, ${ }^{44}$ but also asserted that compelling evidence from observational studies is unlikely. No official initiative has been taken to resolve the contradictions. Given the implications of the negative non-specific effects of DTP for girls, randomised controlled trials (RCTs) of delaying DTP seem justified. ${ }^{45} 46$ The working group on the non-specific effects of vaccines came to similar conclusions, and has ranked an RCT of DTP as a high priority. ${ }^{47}$

Contributors CSB and PA were the chief investigators and are guarantors. CSB, AdR, HW, IML and PA designed the studies. CSB, PA and AdR initiated the studies. AdR, AmR, CSB and PA were responsible for the recruitment and follow-up of participants. BRD, KRL, NL, JR and SBS supervised data collection in different periods. HR was responsible for the statistical analysis and PA wrote the first draft of the paper. All authors contributed to and approved the final version of the paper.

Funding The study was funded by the EU (ICA4-CT-2002-10053), March of Dimes and the Danish National Research Foundation. The Bandim Health Project received support from DANIDA. PA holds a research professorship grant from the Novo Nordisk Foundation.

\section{Competing interests None.}

Ethics approval The protocol was approved by the Danish Central Ethics Committee and the Guinean Ministry of Health's Research Coordination Committee

Provenance and peer review Not commissioned; externally peer reviewed.

\section{REFERENCES}

1. Kristensen I, Aaby P, Jensen H. Routine vaccinations and child survival: follow up study in Guinea-Bissau, West Africa. BMJ 2000;321:1435-8.

2. Aaby $\mathbf{P}$, Jensen $\mathrm{H}$. Routine vaccinations and child survival: effect of gender. BMJ 2002.

3. Breiman RF, Streatfield PK, Phelan M, et al. Effect of infant immunisation on childhood mortality in rural Bangladesh: analysis of health and demographic surveillance data. Lancet 2004;364:2204-11.

4. Vaugelade J, Pinchinat S, Guielle $\mathrm{G}$, et al. Lower mortality in vaccinated children: follow up study in Burkina Faso. BMJ 2004;329:1309-11.

5. Lehmann $\mathbf{D}$, Vail J, Firth MJ, et al. Benefits of routine immunisations on childhood survival in Tari, Southern Highlands Province, Papua New Guinea. Int J Epidemiol 2005; 34:138-48. 
6. Nyarko P, Pence B, Debpuur C. Immunization status and child survival in rural Ghana. Population Council. Working papers No. 147. New York, 2001.

7. Elguero E, Simondon KB, Vaugelade J, et al. Non-specific effects of vaccination on child survival? A prospective study in Senegal. Trop Med Int Health 2005:10:956-60.

8. Moulton LH, Rahmathullah L, Halsey NA, et al. Evaluation of non-specific effects of infant immunizations on early infant mortality in a southern Indian population. Trop Med Int Health 2005;10:947-55.

9. Aaby $\mathbf{P}$, Jensen $\mathrm{H}$, Walraven $\mathrm{G}$. Age-specific changes in the female-male mortality ratio related to the pattern of vaccinations: an observational study from rural Gambia. Vaccine 2006;24:4701-8

10. Aaby $\mathbf{P}$, Jensen $\mathrm{H}$, Rodrigues $\mathrm{A}$, et al. Divergent female-male mortality ratios associated with different routine vaccinations among female-male twin pairs. Int J Epidemiol 2004;33:367-73.

11. Benn CS, Aaby P, Nielsen J, et al. Does vitamin A supplementation interact with routine vaccinations? An analysis of the Ghana vitamin A Supplementation Trial. Am J Clin Nutr 2009;90:629-39.

12. Aaby P, Ibrahim SA, Libman MD, et al. The sequence of vaccinations and increased female mortality after high-titre measles vaccine: trials from rural Sudan and Kinshasa. Vaccine 2006;24:2764-71.

13. Aaby $\mathbf{P}$, Vessari H, Nielsen J, et al. Non-specific and sex-differential effects of routine immunizations in rural Malawi. PIDJ 2006;25:721-7.

14. Jensen H, Benn CS, Lisse IM, et al. Survival bias in observational studies of the impact of routine immunizations on childhood survival. Trop Med Int Health 2007:12:5-14.

15. Aaby P, Benn CS, Nielsen J, et al. Estimating the effect of DTP vaccination on mortality in observational studies with incomplete vaccination data. Trop Med Int HIth 2007;12:15-24.

16. Fine PE, Smith PG. 'Non-specific effects of vaccines'-an important analytical insight, and call for a workshop. Trop Med Int Health 2007;12:1-4

17. Aaby $\mathbf{P}$, Roth $A$, Ravn $H$, et al. Randomized trial of BCG vaccination at birth to low-birth-weight children: beneficial nonspecific effects in the neonatal period? $J$ Infect Dis 2011;204:245-52.

18. Benn CS, Fisker AB, Napirna BM, et al. Vitamin A supplementation and BCG vaccination at birth in low birthweight neonates: two by two factorial randomised controlled trial. BMJ 2010;340:c1101.

19. Martins CL, Garly ML, Balé C, et al. Protective efficacy of standard EdmonstonZagreb measles vaccination in infants aged 4.5 months: interim analysis of a randomised clinical trial. BMJ 2008;337:a661

20. Fine PE, Williams TN, Aaby P, et al. Epidemiological studies of the 'non-specific effects' of vaccines: I-data collection in observational studies. Trop Med Int Health 2009;14:969-76

21. Farrington $\mathbf{C P}$, Firth MJ, Moulton LH, et al.; Working group on non-specific effects of vaccines. Epidemiological studies of the non-specific effects of vaccines: II-methodological issues in the design and analysis of cohort studies. Trop Med Int Health 2009; 14:977-85.

22. Fine PE. Non-specific "non-effects" of vaccination. BMJ 2004;329:1297-8.

23. Valentiner-Branth $\mathbf{P}$, Perch $M$, Nielsen J, et al. Community cohort study of Cryptosporidium parvum infections: sex-differential incidences associated with BCG and diphtheria-tetanus-pertussis vaccinations. Vaccine 2007;25:2733-41.

24. Rodrigues A, Fischer TK, Valentiner-Branth P, et al. Community cohort study of rotavirus and other enteropathogens: are routine vaccinations associated with sex-differential incidence rates? Vaccine 2006;24:4737-46.

25. Huber SA, Pfaeffle B. Differential Th1 and Th2 cell responses in male and female BALB/C mice infected with coxsackievirus group B type 3. J Virol 1994;68:5126-32.

26. Fischer JE, Johnson JE, Johnson TR, et al. Pertussis toxin sensitization alters the pathogenesis of subsequent respiratory syncytial virus infection. $J$ Infect Dis 2000;182:1029-38.
27. Fisker AB, Lisse IM, Aaby $\mathrm{P}$, et al. Impact of neonatal vitamin A supplementation with $B C G$ vaccine on vitamin $A$ status at 6 weeks and 4 months of age. Am J Clin Nutr 2007:86:1032-9.

28. Martins C, Bale C, Garly ML, et al. Girls may have lower levels of maternal measles antibodies and higher risk of subclinical measles infection before the age of measles vaccination. Vaccine 2009;27:5220-5.

29. Diness BR, Martins CL, Balé C, et al. The effect of high-dose vitamin A supplementation at birth on measles incidence during the first 12 months of life in boys and girls: an unplanned study within a randomised trial. $\mathrm{Br} \mathrm{J}$ Nutr 2011:105:1819-22.

30. Veirum JE, Sodemann M, Biai S, et al. Diphtheria-tetanus-pertussis and measles vaccinations associated with divergent effects on female and male mortality at the paediatric ward in Bissau, Guinea-Bissau. Vaccine 2005:23:1197-203.

31. Aaby P, Martins C, Bale C, et al. Sex differences in the effect of vaccines on the risk of hospitalization due to measles in Guinea-bissau. Pediatr Infect Dis J 2010;29:324-8

32. Aaby $\mathbf{P}$, Jensen $\mathrm{H}$, Gomes J, et al. The introduction of diphtheria-tetanuspertussis vaccine and child mortality in rural Guinea-Bissau: an observational study. Int J Epidemiol 2004;33:374-80.

33. Aaby P, Rodrigues A, Biai S, et al. Oral polio vaccination and low case fatality at the paediatric ward in Bissau, Guinea-Bissau. Vaccine 2004;22:3014-17.

34. Aaby $\mathbf{P}$, Jensen $\mathrm{H}$, Garly ML, et al. Routine vaccinations and child survival in a war situation with high mortality: effect of gender. Vaccine 2002;21:15-20.

35. Aaby $\mathbf{P}$, Jensen H, Samb B, et al. Differences in female-male mortality after high-titre measles vaccine and association with subsequent vaccination with diphtheria-tetanus-pertussis and inactivated poliovirus: reanalysis of West African studies. Lancet 2003;361:2183-8.

36. Aaby $\mathbf{P}$, Biai S, Veirum JE, et al. DTP with or after measles vaccination is associated with increased in-hospital mortality in Guinea-Bissau. Vaccine 2007;25:1265-9.

37. Aaby P, Benn CS. Assessment of childhood immunisation coverage. Lancet 2009;373:1428

38. Benn CS, Balé C, Sommerfelt H, et al. Hypothesis: vitamin A supplementation and childhood mortality: amplification of the non-specific effects of vaccines? Int J Epidemiol 2003:32:822-8.

39. Benn CS, Martins C, Rodrigues A, et al. The effect of vitamin A supplementation administered with missing vaccines during national immunization days in GuineaBissau. Int J Epidemiol 2009;38:304-11.

40. Benn CS, Diness BR, Roth A, et al. Effect of 50,000 IU vitamin A given with BCG vaccine on mortality in infants in Guinea-Bissau: randomised placebo controlled trial. BMJ 2008;336:1416-20

41. Aaby P, Martins CL, Garly ML, et al. Non-specific effects of standard measles vaccine at 4.5 and 9 months of age on childhood mortality: randomised controlled trial. BMJ 2010:341:c6495.

42. Roth A, Benn CB, Ravn $\mathrm{H}$, et al. A randomised trial of the effect of revaccination with BCG in early childhood and mortality. BMJ 2010;340:c671

43. Mahieu JM, Muller AS, Voorhoeve AM, et al. Pertussis in a rural area of Kenya: epidemiology and a preliminary report on a vaccine trial. Bull World Health Organ 1978; 56:773-80.

44. Meeting of Global Advisory Committee on Vaccine Safety, 18-19 June 2008 Weekly Epidemiological Record 2008; 83:287-92.

45. Shann F. The non-specific effects of vaccines. Arch Dis Child 2010;95:662-7.

46. Shann F. The nonspecific effects of vaccines and the expanded program on immunization. J Infect Dis 2011;204:182-4.

47. Shann F, Nohynek H. Scott JA, et al.: Working group on nonspecific effects of vaccines. Randomized trials to study the nonspecific effects of vaccines in children in low-income countries. Pediatr Infect Dis J 2010;29:457-61. 